\title{
La cirugía basada en la escasa evidencia: disminución de laparoscopía de emergencia en la era COVID-19
}

\section{Surgery based on scarce evidence: a reduction in emergency laparoscopy in the COVID-19 era}

\section{Correspondencia Yeray Trujillo Loli trujilloyeray@gmail.com}

Recibido: 22/06/2020 Aprobado: 03/09/2020

Citar como: Trujillo-Loli Y, Olivera-Villanueva $M$ Arroyo-Gárate R. La cirugía basada en la escasa evidencia: disminución de laparoscopía de emergencia en la era COVID-19. Acta Med Peru. 2020;37(3):399 401. doi: 10.35663/ amp.2020.373.1013
Yeray Trujillo-Loli1 ${ }^{1,3, a, b}$, Marcia Olivera-Villanuevaa ${ }^{1,3, c}$, Rodrigo Arroyo-Gárate2,3,c

Hospital Nacional Daniel Alcides Carrión. Callao, Perú.

Hospital Nacional Dos de Mayo. Lima, Perú.

Universidad Nacional Mayor de San Marcos. Lima, Perú.

Médico especialista en Cirugía General; ${ }^{b}$ máster en técnicas quirúrgicas endoscópicas; ${ }^{\mathrm{C}}$ médico cirujano

Señor editor,

Desde el inicio de la pandemia se ha investigado cuáles serían las rutas de transmisión del virus SARS-CoV-2, siendo la vía aérea la más aceptada en la actualidad, a través de las partículas virales en forma de aerosol ${ }^{[1]}$. A fines del mes de marzo del 2020, Zhen MH et al. propusieron que era posible el contagio de COVID-19 por medio de la exposición al humo quirúrgico que se producía durante una cirugía laparoscópica ${ }^{[2]}$; basaron su hipótesis en estudios publicados que encontraron otros virus (inmunodeficiencia humana [VIH], hepatitis B [VHB] o papiloma humano [VPH]) en este medio.

A pocos días de esa publicación, las sociedades científicas de cirugía se manifestaron al respecto. La Junta Intercolegial de Cirugía General de Reino Unido e Irlanda recomendó que la cirugía laparoscópica sea reservada para casos seleccionados en donde el beneficio clínico para el paciente excediera sustancialmente al riesgo de transmisión viral ${ }^{[3]}$. La Sociedad Americana de Cirujanos Gastrointestinales y Endoscópicos (SAGES, por sus siglas en inglés) indico poco después que realizar cirugías laparoscópicas era viable, pero bajo ciertas condiciones como el uso de equipos de protección personal (EPP) adecuados, quirófanos a presión negativa, unidades de electrocirugía con la configuración más baja posible, mínimo uso de dispositivos de energía avanzados y el uso de filtros de humo quirúrgico para partículas virales ${ }^{[4]}$.

Estas recomendaciones fueron seguidas por la mayoría de las sociedades científicas de cirugía en el mundo ${ }^{[5]}$; empero, las recomendaciones dadas por la Junta Intercolegial de Cirugía General de Reino Unido e Irlanda fueron criticadas por Mowbray NG et al., catalogándolas como "[...] una respuesta pragmática a la ansiedad generalizada que rodea a la posible transmisión del virus en la sala de operaciones" [6].

Aún no hay evidencia científica que sustente que el virus SARS-Cov-2 pueda transmitirse a través del humo quirúrgico; sin embargo, mientras no se demuestre lo contrario, se deben utilizar filtros de humo. Estos aditamentos deben tener la capacidad de filtrar partículas virales menores de $60 \mathrm{~nm}{ }^{[6]}$ para tener utilidad frente al SARS-CoV-2. Los quirófanos a presión negativa se recomiendan si están disponibles ${ }^{[7]}$ puesto que la mayoría de las salas de operaciones del mundo no cuentan con esta tecnología, sino la característica de tener una presión neutra.

Posteriormente, se retomó la realización de cirugías laparoscópicas e incluso se considera ahora que es la técnica más segura que la cirugía abierta puesto que, a diferencia de esta, se puede controlar la salida del humo quirúrgico mediante el uso de filtros y un sistema de aspiración cerrado, evitando su dispersión por toda la sala de operaciones ${ }^{[5]}$. 
120

100

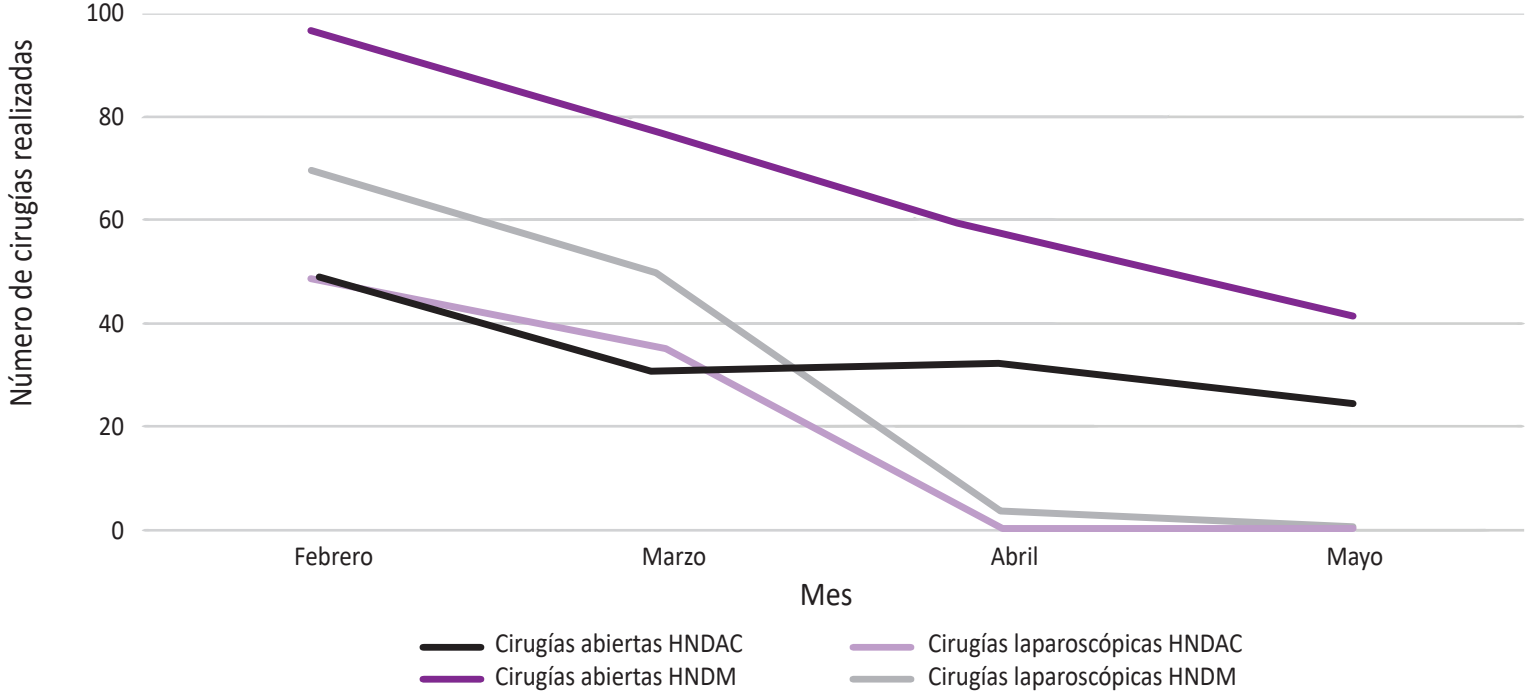

Figura 1. Evolución de cirugías abierta y laparoscópica realizados en el periodo febrero - mayo del año 2020, Hospital Nacional Daniel Alcides Carrión (HNDAC) y Hospital Nacional Dos de Mayo (HNDM)

Durante febrero y marzo se realizaban con regularidad cirugías laparoscópicas en ambos hospitales; desde el inicio de la pandemia (abril), las cirugías laparoscópicas de emergencia fueron casi inexistentes, mientras que las cirugías abiertas continuaron realizándose en menor proporción, por la disminución de la demanda.

Safari S et al. encontraron que la presencia del virus SARS-CoV-2 era inexistente en líquido peritoneal, tejido graso y epiplón ${ }^{[8]}$; tampoco, ocho meses después de la pandemia, se ha reportado que algún integrante del equipo quirúrgico se haya contagiado de COVID-19 por estar expuesto al humo quirúrgico o participar en una cirugía laparoscópica.

En nuestro país, existen hospitales en donde la cantidad de cirugías laparoscópicas de emergencia ha disminuido considerablemente; además, existe oposición de parte del personal de salud a realizarlas, probablemente por temor a contagiarse en sala de operaciones, a pesar de que no existe evidencia científica que respalde esa creencia. Tal es el caso del Hospital Nacional Daniel Alcides Carrión del Callao (HNDAC) en donde en los dos primeros meses de pandemia no se realizaron cirugías laparoscópicas de emergencia (Figura 1), situación parecida a lo ocurrido en el Hospital Nacional Dos de Mayo del Cercado de Lima (HNDM), en donde la disminución también fue considerable.

Estos resultados difieren con lo ocurrido en otros países en donde siguieron realizándose cirugías laparoscópicas. Por ejemplo, Patriti A et al. reportaron en su estudio realizado en Italia que el 69,6\% de las cirugías que se realizaron durante los dos primeros meses de pandemia fueron con abordaje laparoscópico ${ }^{[9]}$. En España, Cano-Valderrama O et al. realizaron un estudio retrospectivo y multicéntrico y encontraron que el $43,3 \%$ de las cirugías realizadas durante la pandemia fueron por laparoscopía ${ }^{[10]}$. En ambos países no se reportaron contagios por exposición al humo quirúrgico.
Por otro lado, dentro de los beneficios de la cirugía laparoscópica se encuentra la menor estancia hospitalaria ${ }^{[11]}$, beneficio que sería especialmente útil durante la pandemia porque en la medida que un paciente permanezca hospitalizado más tiempo, el riesgo de contagiarse por COVID-19 en el hospital será mayor. En el HNDAC, el porcentaje de transmisión por COVID-19 en el servicio de cirugía general, por mayor estancia hospitalaria, fue de 4 de 45 pacientes $(8,8 \%)$, entre los meses de abril y mayo del 2020 , según consta en registros e historias clínicas del hospital.

En una revisión sistemática se evaluó el riesgo de transmisión viral al personal de salud -en el contexto de la pandemia por COVID-19-, comparando la cirugía abierta y la cirugía laparoscópica, y se concluyó que no había datos suficientes para recomendar un abordaje quirúrgico sobre otro ${ }^{[12]}$. En otra revisión que discutía acerca del riesgo de transmisión viral a través del humo quirúrgico durante una cirugía mínimamente invasiva, se concluyó que la evidencia científica disponible no era suficiente para recomendar la suspensión completa de los programas quirúrgicos laparoscópicos y asistidos por robot ${ }^{[13]}$.

Por lo tanto, es importante difundir la evidencia científica que existe sobre este tema porque, a pesar de que en Europa se continúa haciendo cirugía laparoscópica con regularidad, hasta la fecha no se han reportado contagios de COVID-19 por exposición al humo quirúrgico, lo cual nos puede indicar que no hay motivo para no realizar cirugía laparoscópica en pacientes COVID-19, siempre y cuando el equipo quirúrgico tenga el EPP adecuado y cumpla las recomendaciones generales de las sociedades científicas del mundo. 
En la era del COVID-19, nos vimos en la obligación de adaptarnos a la medicina basada en la "escasa" evidencia; empero, esta insuficiente evidencia diseminó el miedo a contagiarse en sala de operaciones, de tal forma que, en nuestro medio, a pesar de que en otros países no hay restricciones respecto a la cirugía laparoscópica, no se ha retomado la práctica de la laparoscopía de emergencia, siendo los pacientes quienes más se perjudican al no beneficiarse con un procedimiento mínimamente invasivo.

Contribuciones de autoría: todos los autores participaron en la concepción y diseño del artículo, su redacción y aprobación de la versión final.

Potenciales conflictos de interés: los autores declaran no tener conflictos de interés.

Fuentes de financiamiento: autofinanciado.

\section{ORCID:}

Yeray Trujillo-Loli, http://orcid.org/0000-0003-2829-5085

Marcia Olivera-Villanueva, http://orcid.org/0000-0001-9662-3592

Rodrigo Arroyo-Gárate, http://orcid.org/0000-0002-8186-0859

\section{REFERENCIAS BIBLIOGRÁFICAS}

1. Morawska L, Cao J. Airborne transmission of SARS-CoV-2: The world should face the reality. Environ Int. 2020;139:105730. doi:10.1016/j. envint.2020.105730.

2. Zheng $M H$, Boni L, Fingerhut A. Minimally invasive surgery and the novel coronavirus outbreak: lessons learned in China and Italy. Ann Surg. 2020;272(1):e5-6. doi:10.1097/SLA.0000000000003924.

3. Royal College of Surgeons of Edinburgh. Intercollegiate General Surgery Guidance on COVID-19 UPDATE [internet]. Edinburgh: The College; 27 de marzo de 2020 [citado 15 de agosto de 2020]. Disponible en: https://www.rcsed.ac.uk/news-public-affairs/ news/2020/march/intercollegiate-general-surgery-guidance-oncovid-19-update
4. Society of American Gastroenterology and Endoscopic Surgeons. SAGES and EAES. Recommendations Regarding Surgical Response to COVID-19 Crisis [Internet]. Los Angeles: The Society; 30 de marzo de 2020 [citado 15 de agosto de 2020]. Disponible en: https://www. sages.org/recommendations-surgical-response-covid-19

5. Balibrea J, Badia J, Rubio I, Martín E, Álvarez E, García S, et al. Manejo quirúrgico de pacientes con infección por COVID-19. Recomendaciones de la Asociación Española de Cirujanos. Cir Esp. 2020;98(5):251-9. doi:10.1016/j.ciresp.2020.03.001.

6. Mowbray NG, Ansell J, Horwood J, Cornish J, Rizkallah P, Parker A et al. Safe management of surgical smoke in the age of COVID-19. Br J Surg. 2020;10.1002/bjs.11679. doi:10.1002/bjs.11679.

7. Steward JE, Kitley WR, Schmidt CM, Sundaram CP. Urologic Surgery and COVID-19: how the pandemic is changing the way we operate. J Endourol. 2020;34(5):541-9. doi:10.1089/end.2020.0342.

8. Safari S, Keyvani H, Malekpour N, Dehghanian A, Razavi H, Nemati $B$, et al. Abdominal surgery in patients with COVID-19: detection of SARS-CoV-2 in abdominal and adipose tissues. Ann Surg. 2020;272(3):e253-6. doi: 10.1097/SLA.0000000000004165

9. Patriti A, Baiocchi GL, Catena F, Marini P, Catarci M; FACS on behalf of the Associazione Chirurghi Ospedalieri Italiani (ACOI). Emergency general surgery in Italy during the COVID-19 outbreak: first survey from the real life. World J Emerg Surg. 2020;15(1):36. doi:10.1186/ s13017-020-00314-3.

10. Cano-Valderrama $\mathrm{O}$, Morales $\mathrm{X}$, Ferrigni $\mathrm{CJ}$, Martin-Antona $\mathrm{E}$, Turrado V, García A, et al. Acute care surgery during the COVID-19 pandemic in Spain: changes in volume, causes and complications. A multicentre retrospective cohort study. Int J Surg. 2020;80:157-61. doi:10.1016/j.ijsu.2020.07.002

11. Ohtani H, Tamamori Y, Arimoto Y, Nishiguchi Y, Maeda K, Hirakawa K. Meta-analysis of the results of randomized controlled trials that compared laparoscopic and open surgery for acute appendicitis. J Gastrointest Surg. 2012;16(10):1929-39. doi:10.1007/s11605-012-1972-9.

12. Patterson TJ, Currie PJ, Beck J, Spence RAJ, Spence GM. A systematic review of viral transmission risk to healthcare staff comparing laparoscopic and open surgery. Surgeon. 2020;S1479666X(20)30088-3. doi:10.1016/j.surge.2020.06.016.

13. Pavan N, Crestani A, Abrate A, de Nunzio C, Esperto F, Giannarini $G$, et al. Risk of Virus contamination through surgical smoke during minimally invasive surgery: a systematic review of the literature on a neglected issue revived in the COVID-19 pandemic era. Eur Urol Focus. 2020;6(5):1058-69. doi:10.1016/j.euf.2020.05.021. 